\title{
Study on Driving Performance of the Axial-Flow Blood Pump under the Condition of Large Gap
}

\author{
Yan Xu, Lizhi Cheng, and Liang Liang \\ Department of Mechanical \& Electrical Engineering, Changsha University, Changsha 410003, China \\ Correspondence should be addressed to Yan Xu; x_y616@163.com
}

Received 1 October 2016; Revised 22 December 2016; Accepted 10 January 2017; Published 13 February 2017

Academic Editor: Hyeong Joon Ahn

Copyright (C) 2017 Yan Xu et al. This is an open access article distributed under the Creative Commons Attribution License, which permits unrestricted use, distribution, and reproduction in any medium, provided the original work is properly cited.

\begin{abstract}
The paper demonstrates an improvement of the simulation and computational methods for research on the system magnetic field and driving performance of the large gap magnetic drive system, which is used to drive the axial flow blood pump. The operational principle and structure of large gap magnetic drive system are narrated. Ansoft is adopted to simulate a three-dimensional driving torque to improve accuracy of computation. Experiments and theoretical study show that the use of Z10-T25 oriented silicon steel sheets as the electromagnetic core material can remarkably improve the system driving performance as well as optimize the volume and weight of the electromagnets. So the electromagnet made with oriented silicon steel sheets is conducive to improving the driving performance.
\end{abstract}

\section{Introduction}

With the development of biomedical engineering, the use of artificial heart (blood pump) has become the main way to save the patients with heart failure. The blade type artificial heart (blood pump) can be divided into axial-flow and centrifugal type. The axial-flow blood pump has the advantages of small volume, being easy to implant, and less damage to blood. The magnetic performance of electromagnet is the major factor that decides the system driving performance when large gap magnetic drive system is applied to the driving of axial-flow blood pump [1].

In the magnetic field optimization method and performance evaluation research field, Aminfar et al. [2] have studied the behavior of a two-dimensional tube with an elastic segment containing ferrofluid, in presence of nonuniform magnetic field. Two cases of magnetic field including constant gradient (both positive and negative) and field of a wire, carrying electric current, were examined. Numerical solution of governing equations of the flow field has been obtained. Aghaei et al. [3] have investigated the effects of magnetic field on the flow field, heat transfer, and entropy generation of $\mathrm{Cu}$ water nanofluid mixed convection in a trapezoidal enclosure. The finite volume method and SIMPLER algorithm have been utilized to solve the governing equations numerically.
In order to get stable mechanical output performance of giant magnetostrictive actuator, with the GMM rod $(10 \times 100 \mathrm{~mm})$ as the object of the research, Yu et al. [4] have established the finite element model of magnetic field intensity distribution for drive coil and permanent magnetic cylinder. By using ANSYS Maxwell software, the influence of average magnetic field intensity and magnetic field uniformity on its axis from different parameters have been obtained. Deng et al. [5] have proposed a zero-dimensional model to analyze the magnetic flux compression process of a thin cylindrical liner driven by electromagnetic force. The magnetic energy conversion efficiency and 3 optimal dimensionless parameters in the magnetic flus compression process are obtained. Lee et al. [6] have developed and evaluated the performance of a new magnetic bearing system having a motor for a new thirdgeneration blood pump. The blood pump is driven by an integration motor. The gap between the rotor and the stator is $0.2 \mathrm{~mm}$. Jiao and Liu [7] have analyzed the requirements of the intensity and distribution of cusp magnetic flux density to control the convection and proposed the optimization design goal. The simulation model of cusp magnetic field is built by the finite element with three-dimension (3D) modeling method and the magnetic field structure was optimized. Zheng et al. [8] have studied the force and torque performance for magnetically driven blood pump. The 
magnetic field simulations for three different magnetic coupling arrangements are obtained numerically. And the pump performance is obtained experimentally. Lekhchine et al. [9] present the analysis of performances of speed fuzzy logic controller. The simulation results show that the fuzzy logic controller ensures the best dynamic performances in rotor resistance and load variations. Kül et al. [10] have analyzed and simulated the low power line start permanent magnet synchronous motor (LSPMSM) using finite element method using ANSYS RMXPRT and Maxwell modeling software. Parameters of three motors are changed, are modeled, and then put to the simulation. The torque and efficiency curve are acquired and compared with each other. The results are evaluated in terms of feasibility for the industrial area in terms of efficiency and torque.

On the basis of the above research results about magnetic field optimization method and performance evaluation, this study has optimized the driving performance of electromagnet from the magnetic field simulation method and the materials and structural parameters of the electromagnet. The relevant research results provide more accurate and reliable guide for the design of large gap magnetic drive system to increase the system driving performance.

\section{Operational Principle and Structure of Large Gap Magnetic Drive System}

2.1. The Structure and Physical Principle of the Axial-Flow Blood Pump. The axial-flow blood pump is composed of bearings, an impeller, and a permanent magnet, as shown in Figure 1. The bearings of the current axial-flow blood pump are ceramic bearings because they have good corrosion resistance and are used in the field of medical equipment normally. In the experimental research of this paper, part of the system energy loss is due to bearing friction. In our future studies, the new structure of axial-flow blood pump will be improved by adopting magnetic suspension bearing. Then the new axial-flow blood pump can avoid thrombosis and destruction of blood cell and be induced by friction effectively. This is an improved direction of our researches.

By coupling between the permanent magnet and the other magnetic field, the permanent magnet and the impeller can be driven and rotated. The photo of axial-flow blood pump is shown in Figure 10(a).

\subsection{The Working Principle of the Large Gap Magnetic Drive} System. The magnetic drive system structure is shown in Figure 2, including the electromagnet and the permanent magnet rotor. The electromagnet is composed of four coils and two T-shape iron cores which are superimposed by the silicon steel sheet. The electromagnet is shown in Figure 10(b). The permanent magnet is a cylindrical permanent magnet and magnetized in radial direction. It can be embedded in the axial-flow blood pump rotor shown in Figure $1 . h$ is the pole gap that reaches $20 \mathrm{~mm}$.

In the technical project proposed by the paper, the magnetic system is divided into two parts. One part is the axialflow blood pump comprising permanent magnet; the other

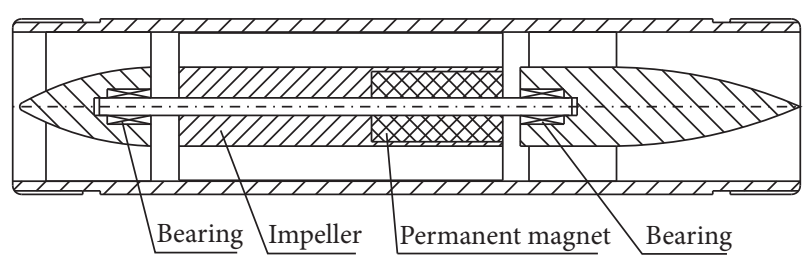

FIGURE 1: Schematic of axial-flow blood pump.

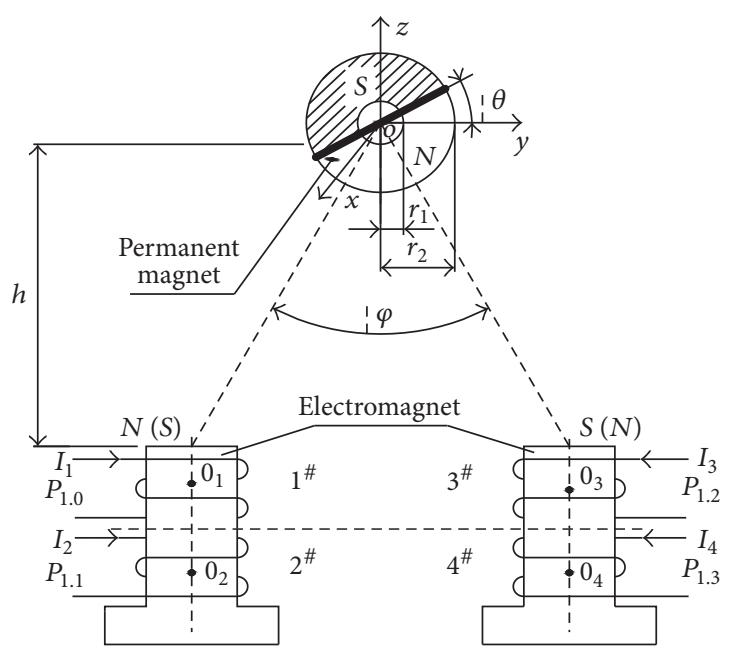

FIGURE 2: Schematic diagram of magnetic drive system.

one is electromagnet driving device. The axial-flow blood pump is implanted into human body and connected with the heart aorta. The electromagnet driving device is placed out of human body. The large gap magnetic drive system transfers energy by magnetic field coupling between electromagnet and permanent magnet noncontact. The technical project has a good application prospect because it does not need the wire going through the patients' skin. So patients' pain can be decreased to a minimum.

As shown in Figure 2, the iron core is circled by 4 groups of coils. These coils output pulses through the MCU, as shown in Figure 4. P1.0 causes coil $1^{\#}$ to produce N pole on the left side of the permanent magnet; P1.1 causes coil $2^{\#}$ to produce $S$ pole on the left side of the permanent magnet; P1.2 causes coil $3^{\#}$ to produce $S$ pole on the right side of the permanent magnet, and $\mathrm{P} 1.3$ causes coil $4^{\#}$ to produce $\mathrm{N}$ pole on the right side of the permanent magnet. So all kinds of electromagnet magnetic poles' state, as shown in Figure 3, can be realized and the permanent magnet can be driven by the control software.

The system drive torque is generated by the couple effect between electromagnet field which is energized by coils and permanent magnet field. The load torque is generated by the load on the axial blood pump impeller. The permanent magnet rotor rotates $360^{\circ}$ with that electromagnet and completes the variation of SS $\rightarrow \mathrm{NS} \rightarrow \mathrm{NN} \rightarrow \mathrm{SN}$ [11]. The permanent magnet's speed can be adjusted by modifying the electromagnet's magnetic poles' state switching frequency. Furthermore, by modifying the timing of the electromagnet's 


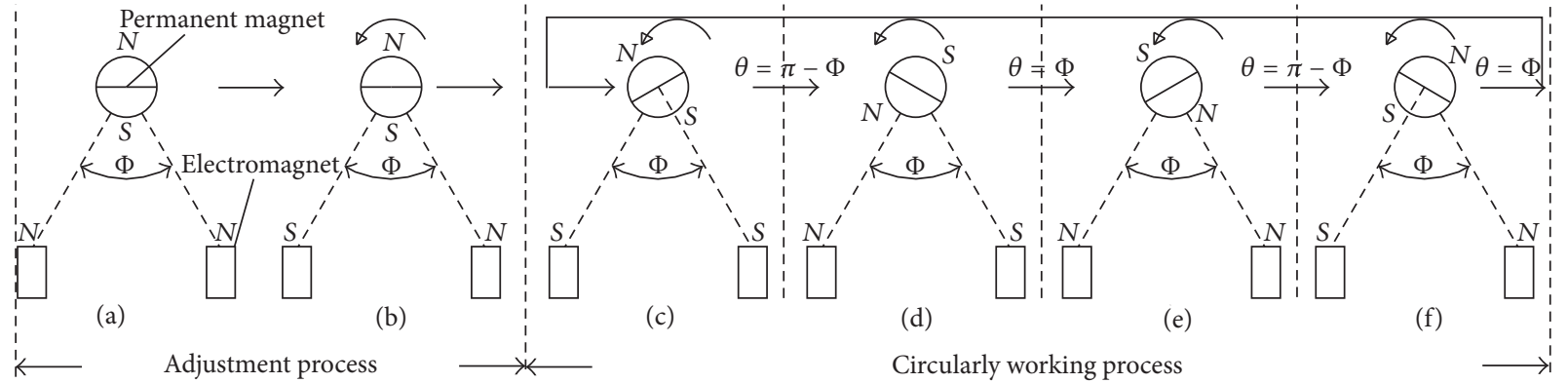

FIgURE 3: Principle of rotation of the passive permanent magnet.

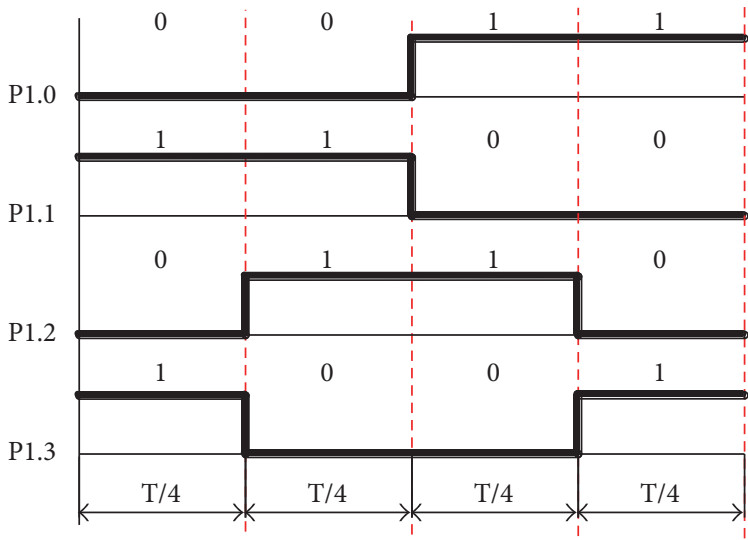

Figure 4: Output pulse succession of electromagnet's coil.

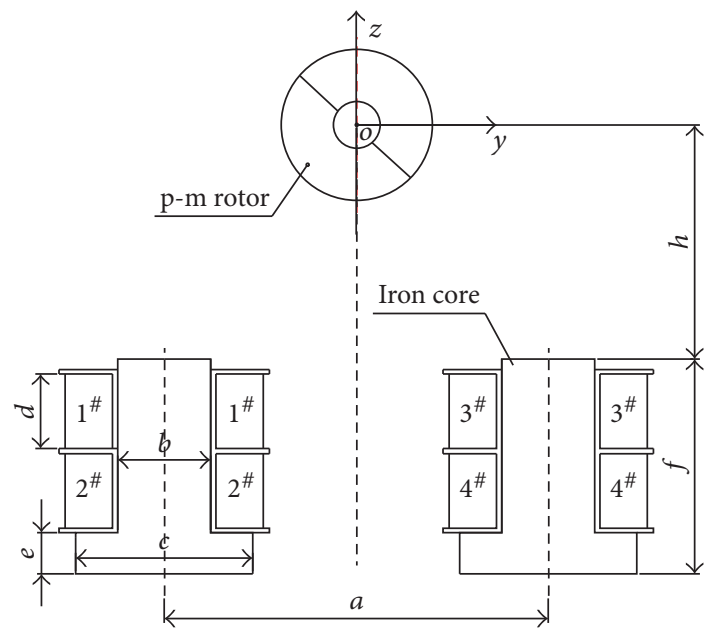

FIGURE 5: The electromagnet iron core structure of magnetic drive system.

magnetic poles state switching, it is clear that there is a clockwise rotation of the permanent magnet $(\mathrm{p}-\mathrm{m})$.

\subsection{The Electromagnet Iron Core Structure and Naming} Method. The electromagnet iron core of magnetic drive system has a double $\mathrm{T}$ structure, as shown in Figures 2 and 5. In Figure 5, $1^{\#}, 2^{\#}, 3^{\#}$, and $4^{\#}$ represent four coils of the electromagnet, $h$ is the magnetic pole coupling distance between electromagnet and the p-m rotor, $a$ is the center distance between the two poles of the electromagnet and the dimensions of the other parts of the iron core, which are indicated by $b, c, e$, and $f$ in the diagram, and $d$ represents the coil height. The parameters $h$ and $a$ can be adjusted. The silicon steel sheets used are of common transformer and have been fabricated as iron core of the electromagnet. The selectable structures and dimensions have certain limitations because of the limitations of raw materials.

The electromagnet iron core is named according to dimension $c$, that is, "material + shape + size," as indicated in the diagram. The size of the double $\mathrm{T}$ electromagnet is $c$ $=34 \mathrm{~mm}$ and the material is DW-310-35 (simplified as D35) silicon steel sheet; the iron core is named as D35-T34.

\section{Study on Driving Performance of the Axial-Flow Blood Pump}

The driving performance of the axial-flow blood pump is the driving capability between the active electromagnet and driven permanent magnet under the condition of the definite system structure parameters and magnetic pole coupling distance $h$. The main factor of system driving performance is the driving torque which is acted on the permanent magnet by magnetic field coupling. In order to improve the system driving performance under the premise of the same structural parameters, the current of coil should be increased in this paper. With the increase of the current of coil, the power consumption and the temperature of coil and electromagnet iron core are gradual increasing. In order to improve the efficiency of power transfer, the minimum power consumption method is studied [11]. The temperature distribution, heat loss, heat flux, and thermal gradient of various components of the system were analyzed, and the temperature rise of coil and electromagnet iron core can be effectively reduced by improving the heat emission condition of the electromagnet [12]. In our future studies, the relationship between system driving performance and the system power consumption and the temperature of coil and electromagnet iron core will be included and promoted.

3.1. Improvement on Magnetic Field Simulation Method. Our preliminary research simplifies the system into a twodimensional finite element model for modeling, in which 
ANSYS software is adopted to simulate and calculate the system driving torque to obtain the magnitude of driving torque for the unit length of the permanent magnet. According to the axial length of the permanent magnet, the two-dimensional driving torque proximity transfers into the three-dimensional driving torque through the equivalent formula: (two-dimensional driving torque) $\times$ (axial length of the permanent magnet $)=$ three-dimensional driving torque.

According to our previous research results [11], the system driving torque calculation model can be expressed as

$$
\begin{aligned}
T= & I \cdot \frac{2 \mu N D M}{\cos \theta} \\
& \cdot \int_{-l / 2}^{l / 2}\left[\int_{r_{2} \cos \theta}^{r_{1} \cos \theta}\left(y \cdot B_{y}^{\prime}+y \cdot B_{z}^{\prime} \tan \theta\right) d y\right] d x,
\end{aligned}
$$

where $T$ is the system driving torque $(\mathrm{N} * \mathrm{~mm}) . \mu$ is used to build the system spatial magnetic field calculation model. It is the magnetic permeability of the medium which energized the space where the coil is. Its value is changed with the magnetic permeability curve. $N$ and $D$ are the number of windings and the area of coil's projection in xoy coordinate plane, respectively. $\theta$ is the angle between the magnetic pole of the permanent magnet line and the $y$-axis. $r_{1}, r_{2}, l$, and $M$ are the inner radius, the outer radius, the axial length, and magnetization of the permanent magnet, respectively. $B_{y}$ and $B_{z}$ are the spatial magnetic field components in the $y$-axis and $z$-axis directions at the points $(x, y, z)$, respectively. $B_{y}^{\prime}$ and $B_{z}^{\prime}$ are the factor of $B_{y}$ and $B_{z}$.

The system driving torque can be computed by MATLAB according to formula (1). In this paper, the computation result obtained in MATLAB is taken as a standard; then the threedimensional driving torque obtained in Ansoft and twodimensional driving torque obtained in ANSYS are compared with the standard. So the computation deviation of $3 \mathrm{D}$ and $2 \mathrm{D}$ simulation relative to the standard can be quantified.

The magnetic field simulation method is improved in this study, and Ansoft is adopted to simulate, analyze, and calculate the three-dimensional driving torque. The system driving torque values obtained by the two methods are compared with the computation result obtained by using MATLAB. The magnetic simulation method has been improved to obtain more accurate and reliable research results.

3.1.1. Building of the Three-Dimensional Model. The threedimensional simulation model of the magnetic drive system is shown in Figure 6. With regard to the simulation and analysis of the driving torque of the current electromagnet D35-T34, the structure and electromagnetic parameters are provided below (the values of $a, b, c, d, e$, and $f$ are indicated in Figure 5).

In the paper, the Solution Type of Ansoft Maxwell 3D is magnetostatic. The boundary conditions are default of the software, and the meshing method is length based. The cell size of permanent magnet is set as 0.002 , and the other cell size is set as 0.01 . The current loads are applied across the sections of four coils, respectively. The current loads are $N I=$ 585.

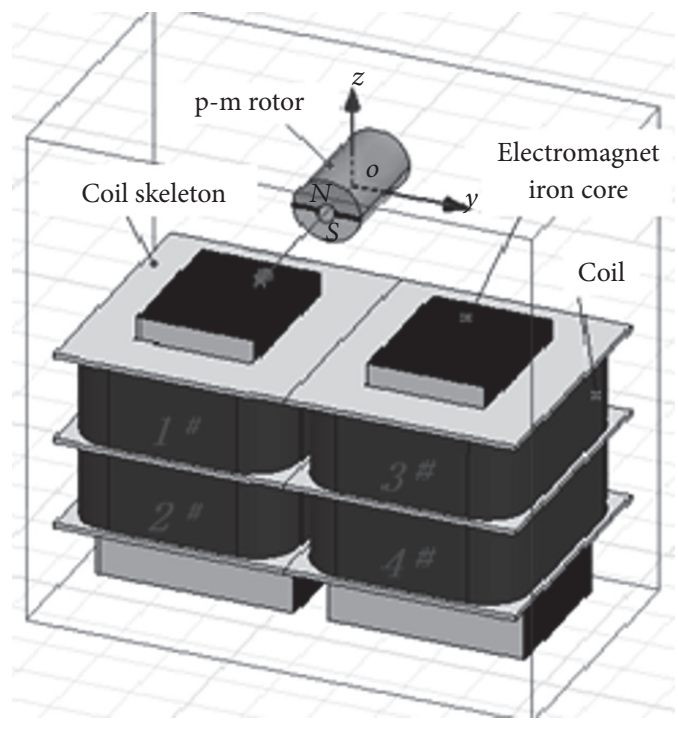

FIGURE 6: The three-dimensional simulation model of the magnetic drive system.

The relative permeability of electromagnet is 5.475 . The coercivity $H c$ of permanent magnet is $900 \mathrm{KA} / \mathrm{m}$, and its relative permeability is 1.087 . The coil material is set as copper, and the other one is set as vacuum.

The structural parameters of the electromagnet iron core are $b=22 \mathrm{~mm}, c=34 \mathrm{~mm}, e=14 \mathrm{~mm}$, and $f=54 \mathrm{~mm}$. It is made of laminated $\mathrm{T}$ silicon steel sheets with thickness of $0.35 \mathrm{~mm}$. In building the electromagnet simulation model, 71 pieces of silicon steel sheets are used, with the clearance between the sheets being $0.007 \mathrm{~mm}$. The lamination parameter of silicon steels sheet is 0.97 , and the coil height is $d=$ $15 \mathrm{~mm}$.

Parameters of the p-m rotor: the inner diameter is $2 \mathrm{~mm}$, the outer diameter is $9 \mathrm{~mm}$, and the axial length is $15 \mathrm{~mm}$.

Relative position parameters are $a=44 \mathrm{~mm}$ and $h=$ $20 \mathrm{~mm}$.

By changing the current loading state of the simulation model, the simulated electromagnet generates 4 magnetic pole states, namely, SS, NS, NN, and SN. By changing the coercive force of the $\mathrm{p}-\mathrm{m}$ rotor, the corresponding angular displacement of the $\mathrm{p}-\mathrm{m}$ rotor is simulated during its turning for one circle. Subsequently, simulation analysis of the magnetic torque during the turning is conducted to obtain the three-dimensional driving torque value under different magnetic pole coupling distances.

\subsubsection{Magnetic Field Simulation and Comparative Analysis.} The magnetic pole coupling distance $h$ is adjusted to 20, $30,40,50$, and $60 \mathrm{~mm}$; then $3 \mathrm{D}$ Ansoft simulation method and 2D ANSYS simulation method are used to calculate the average driving torques of the blood pump p-m rotor that has been turning for one circle under different conditions. A comparative analysis of the above two simulation results with the analytic value of MATLAB values is then conduced, and the deviation between the above simulation results and the numerical calculation is determined. The relevant data are 
TABLE 1: Theory and simulation result of average driving torque.

\begin{tabular}{|c|c|c|c|c|c|c|}
\hline Number & $h(\mathrm{~mm})$ & 2D ANSYS $(\mathrm{N} \mathrm{mm})$ & 3D Ansoft $(\mathrm{N} \mathrm{mm})$ & MATLAB value $(\mathrm{N} \mathrm{mm})$ & ANSYS deviation (\%) & Ansoft deviation (\%) \\
\hline 1 & 20 & 2.252 & 2.096 & 1.941 & 16.4 & 7.9 \\
\hline 2 & 30 & 1.888 & 1.661 & 1.510 & 25.1 & 11.1 \\
\hline 3 & 40 & 1.506 & 1.364 & 1.186 & 27.9 & 14.6 \\
\hline 4 & 50 & 1.310 & 1.096 & 0.970 & 34.6 & 13.4 \\
\hline 5 & 60 & 1.004 & 0.876 & 0.755 & 32.8 & 16.1 \\
\hline Average & - & - & - & - & 27.4 & 12.6 \\
\hline
\end{tabular}

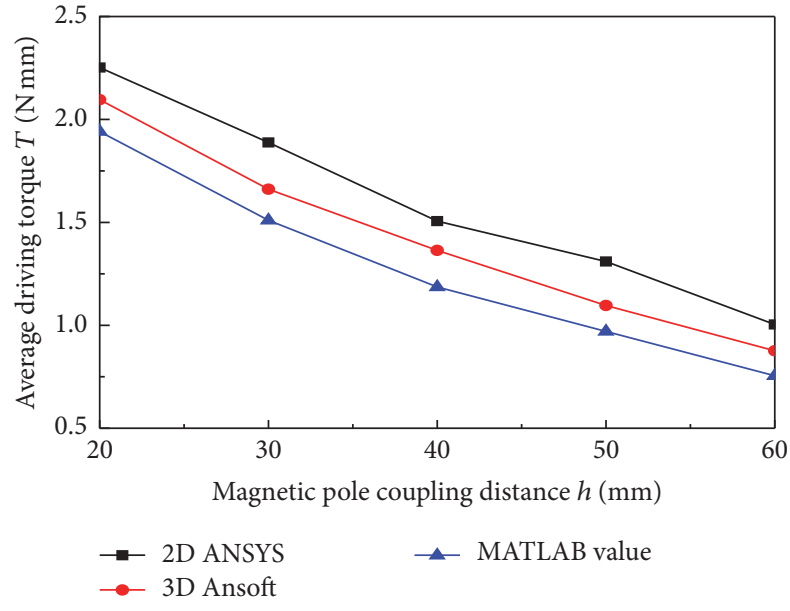

FIGURE 7: Theory and simulation results comparison of average driving torque.

shown in Table 1, and the relation curves of changes of the three results with the magnetic pole coupling distances are shown in Figure 7.

Figure 7 shows that the equivalent driving torque obtained from the two-dimensional ANSYS simulation method considerably differs from the result of numerical calculation; the maximum deviation has reached $34.6 \%$ and the average deviation is up to $27.4 \%$. The average deviation of results obtained using Ansoft for magnetic field threedimensional modeling analysis compared with the numerical calculation is reduced to $12.6 \%$. When the coupling distance is $20 \mathrm{~mm}$, the minimum deviation of $7.9 \%$ is obtained between the results of Ansoft three-dimensional modeling and MATLAB numerical calculation.

Hence, the three-dimensional modeling simulation with Ansoft can obtain calculation results of higher accuracy compared with two-dimensional calculation. The average deviation of the Ansoft result is basically within the acceptable range. Thus, Ansoft Maxwell 3D is adopted to conduct finite element simulation for the driving performance of large gap magnetic drive system to improve the magnetic field simulation method.

\subsection{Improvement of Materials and Structural Parameters of Electromagnet}

3.2.1. Comparison of the Electromagnet Iron Cores. As the medium for system electromagnetic energy conversion, electromagnet plays an important role in improving the driving performance of a magnetic drive system. Such improvement can be realized if less electricity is used for obtaining larger magnetic energy. In addition, selection of electromagnet core material is critical to the whole magnetic drive system. The electromagnet core material currently used is nonoriented silicon steel sheet DW310-35 (simplified as D35), which is formed by lamination. Given that the iron loss and permeability of nonoriented silicon steel have different characteristics, the magnetic field in the direction of connection of the electromagnet and blood pump p-m rotor generates driving action, while the magnetic fields generated in other directions do not have any action. Oriented silicon steel is characterized by low iron loss and high permeability, and its rolling direction has higher permeability and lower iron loss than other directions. If its rolling direction is oriented to the direction of connection of blood pump p-m rotor, which utilizes the higher permeability and lower iron loss, then the system magnetic performance may increase effectively.

Therefore, this study adopts the oriented silicon steel sheet 27ZH100 (simplified as Z10) as the electromagnet iron core material to optimize the driving performance of the large gap magnetic drive system.

\subsubsection{Simulation of the Driving Performance of Electromag-} nets with Different Materials and Structural Parameters. The model is built according to the structural dimensions and parameters of the electromagnet iron core fabricated with the transformer iron core; the material is then used as object for experimental research. The experimental results are compared with the 3D Ansoft modeling simulation result to determine the best electromagnetic material and structural parameters for the driving performance of large gap magnetic drive system.

(1) Building of Simulation Model. According to the current experimental conditions, the parameters of the electromagnet iron core for the simulation model are provided below:

D35-T34 Electromagnet Iron Core. The material is D35 silicon steel sheet with dimensions of $a=44 \mathrm{~mm}$ which is the minimum value of D35-T34, $b=22 \mathrm{~mm}$, $c=34 \mathrm{~mm}, d=15 \mathrm{~mm}, e=14 \mathrm{~mm}$, and $f=$ $54 \mathrm{~mm}$. The thickness of a single sheet is $0.35 \mathrm{~mm} ; 71$ pieces of silicon steel sheets are used for modeling. The clearance between the sheets is $0.007 \mathrm{~mm}$, and the total thickness of iron core is approximately $25 \mathrm{~mm}$. 


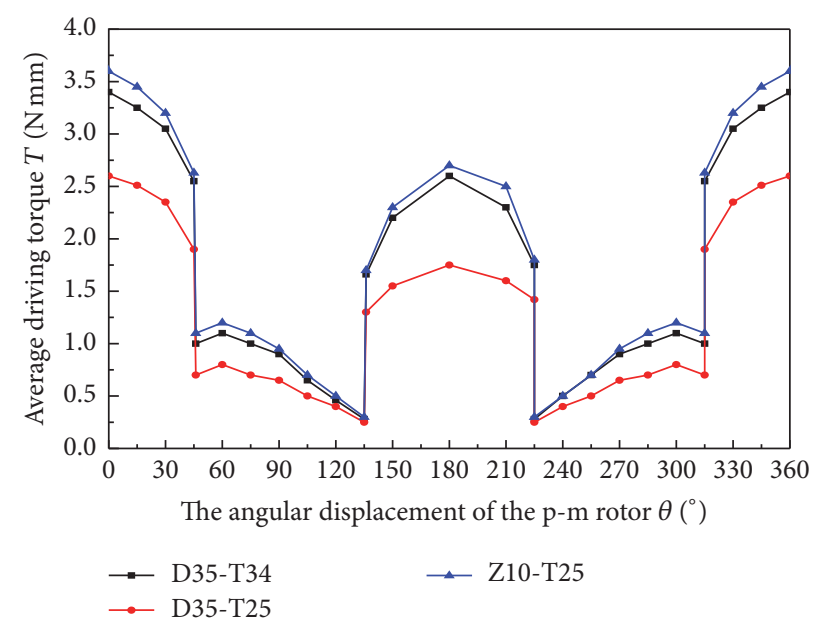

Figure 8: Comparison of driving torque simulation envelope of three kinds of electromagnet.

D35-T25 Electromagnet Iron Core. The material is D35 silicon steel sheet with dimensions of $a=36 \mathrm{~mm}$ which is the minimum value of D35-T25, $b=19 \mathrm{~mm}$, $c=25 \mathrm{~mm}, d=14 \mathrm{~mm}, e=9 \mathrm{~mm}$, and $f=$ $38 \mathrm{~mm}$. The thickness of a single sheet is $0.35 \mathrm{~mm} ; 65$ pieces of silicon steel sheets are used for modeling. The clearance between the sheets is $0.007 \mathrm{~mm}$, and the total thickness of iron core is approximately $23 \mathrm{~mm}$.

Z10-T25 Electromagnet Iron Core. The material is Z10 silicon steel sheet with the same dimensions as D35T25. For example, $a=36 \mathrm{~mm}$ is the minimum value of D35-T25. As the thickness of a single sheet is $0.27 \mathrm{~mm}, 83$ pieces of silicon steel sheets are used for modeling. The total thickness of the iron core is the same as that of D35-T25. The clearance between the sheets is $0.007 \mathrm{~mm}$, and the total thickness of iron core is approximately $23 \mathrm{~mm}$.

The parameters of the other structures are as follows: the inner and outside diameters of $\mathrm{p}-\mathrm{m}$ rotor are $2 \mathrm{~mm}$ and $9 \mathrm{~mm}$, respectively; the axial length is $15 \mathrm{~mm}$ and the magnetic pole distance is $h=20 \mathrm{~mm}$.

(2) Simulation and Comparative Analysis of Driving Performance. According to the operational principle of a large gap magnetic drive system, when the output state of electromagnetic pole is NS, the change range of the angular displacement of the p-m rotor $\theta_{1}$ is $\left[0,45^{\circ}\right]$ and $\left[315^{\circ}, 360^{\circ}\right]$. Furthermore, when the output state of electromagnetic pole is $\mathrm{NN}$, the change range of the angular displacement of the p-m rotor $\theta_{2}$ is $\left[45^{\circ}, 135^{\circ}\right]$. When the output state of electromagnetic pole is $\mathrm{SN}$, the change range of the angular displacement of the $\mathrm{p}-\mathrm{m}$ rotor $\theta_{3}$ is $\left[135^{\circ}, 225^{\circ}\right]$. Finally, when the output state of electromagnetic pole is SS, the change range of the angular displacement of the $\mathrm{p}-\mathrm{m}$ rotor $\theta_{4}$ is $\left[225^{\circ}, 315^{\circ}\right]$.

The maximum driving torque values generated by the operation of blood pump p-m rotor in one circle are given in Figure 8 . The values are obtained by simulation analysis of the

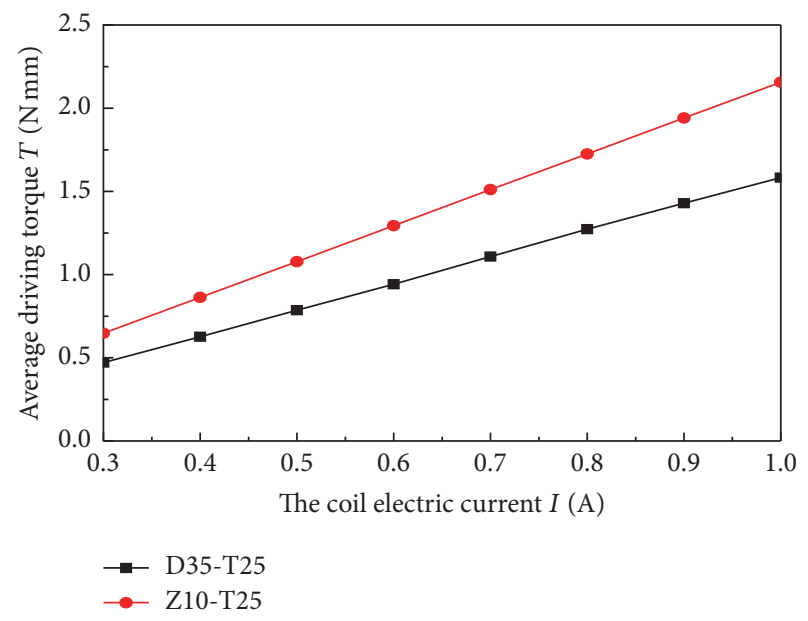

FIGURE 9: Average magnetic driving torque of two kinds of electromagnet with the coil current changes.

driving torque generated by the three kinds of electromagnet when the electric current of a single coil is $0.9 \mathrm{~A}$.

Figure 8 indicates the following:

(1) For the same dimensions and electromagnetic input parameters, the electromagnet Z10-T25 that has used oriented silicon steel sheets obviously generates larger magnetic driving torque compared to D35-T25.

(2) For the same electromagnetic materials, the D35-T34 with larger structural dimensions generates stronger magnetic driving torque than D35-T25.

(3) For the same electromagnetic parameters, the electromagnet Z10-T25 generates slightly higher driving torque than the electromagnets made with nonoriented silicon steel core (i.e., D35-T34), although the structural dimensions of D35-T34 are slightly smaller than those of Z10-T25.

Simulation analyses are conducted for electromagnets D35T25 and Z10-T25 of similar structures and dimensions but made with different materials to obtain the law of effect of the coil electric current on the average driving torque during its turning for one circle.

Figure 9 shows that, under the same structure and electromagnetic parameters, when Z10 oriented silicon steel sheets are used as electromagnet iron core, the generated driving torque average value is $36.7 \%$ higher than D35 nonoriented silicon steel sheets. In addition, the increase of magnetic performance of the Z10 iron core is larger than that of the D35 iron core with the continual increase of current. Hence, the use of Z10-T25 oriented silicon steel sheets as electromagnet iron core can increase the magnetic driving performance of the system.

3.3. Experimental Research on the Driving Performance of the Axial-Flow Blood Pump. Based on the above results on driving performance, the corresponding electromagnets are applied to the experiments of axial-flow blood pump driving to compare their driving torque according to the 


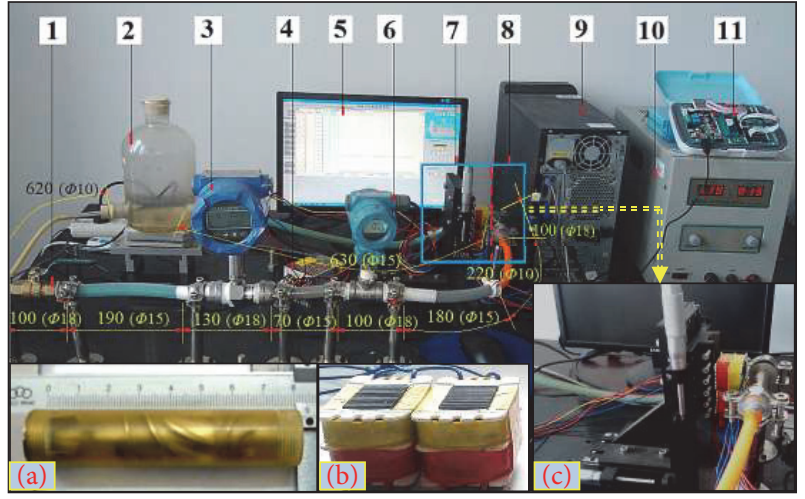

FIGURE 10: Experiment Device. 1: damping valve; 2: water reservoir; 3: eddy flow sensor; 4: $12 \mathrm{~V}$ switching power supply; 5 : monitoring system; 6: pressure transmitter; 7: electromagnet and mobile platform; 8: axial-flow blood pump; 9: PC; 10: DC power source; 11: SCM control system.

limit of rotating speed that can be reached by different electromagnets.

3.3.1. Introduction of Experimental Equipment. According to our previous research results [11], a relatively simple experiment system is built and some experiments on the system dynamic characteristics were performed. On this basis, now the experimental condition is equipped with a monitoring system, eddy flow sensor, and a pressure transmitter. Its accuracy and real-time performance are better. The experiment device contains a damping valve (1), a glycerolwater mixture (blood analog) reservoir (2), an eddy flow sensor (3), $12 \mathrm{~V}$ switching power supply (4), a monitoring system (5), a pressure transmitter (6), an electromagnet and mobile platform (7), an axial-flow blood pump (8), a PC (9), a DC power source (10), and SCM control system (11), as shown in Figure 8. The axial-flow blood pump is shown in Figure 10(a). The electromagnet is shown in Figure 10(b). The mobile platform is shown in Figure 10(c). The eddy flow sensor is LWGY- $15 \mathrm{Cl}$ and its accuracy is $0.001 \mathrm{~m}^{3} / \mathrm{h}$. The pressure transmitter is OT-V80 and its accuracy is $0.01 \mathrm{KP}$.

3.3.2. Electromagnets Used for Experiments. The material objects of the D35-T34, D35-T25, and Z10-T25 electromagnets are shown in Figure 11.

In Figure 11, (a) and (b) are electromagnets made with laminated D35 nonoriented silicon steel sheets and Z10 oriented silicon steel sheets, which are of the same structural dimensions; (c) is the electromagnet made with laminated D35 nonoriented silicon steel sheets.

The working conditions of experimental data in Table 2: The electromagnets are D35-T34, D35-T25, and Z10-T25, respectively. For comparison purposes, the parameter $a$ of the three electromagnets is adjusted to $44 \mathrm{~mm}$. The other parameters of three electromagnets are the same as the parameters of the above-mentioned simulation modeling in this paper. The coupling distances $h$ of the electromagnetic poles with the p-m rotor are $20 \mathrm{~mm}, 30 \mathrm{~mm}, 40 \mathrm{~mm}, 50 \mathrm{~mm}$,

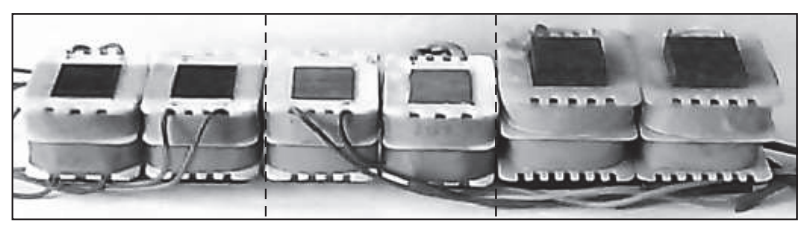

(a) D35-T25

(b) Z10-T25

(c) D35-T34

FIGURE 11: The material objects of electromagnets.

TABLE 2: The maximum speed of blood pump under the condition of different electromagnet iron core.

\begin{tabular}{lrrrrr}
\hline$h(\mathrm{~mm})$ & 20 & 30 & 40 & 50 & 60 \\
\hline $\begin{array}{l}\text { The rotating speed of blood } \\
\text { pump } n(\mathrm{r} / \mathrm{min})\end{array}$ & & & & & \\
D35-T34 & 8400 & 6600 & 5300 & 4200 & 3200 \\
D35-T25 & 7800 & 6200 & 4900 & 3900 & 2800 \\
Z10-T25 & 8600 & 6800 & 5400 & 4300 & 3200 \\
\hline
\end{tabular}

and $60 \mathrm{~mm}$, respectively. The output current of the DC power source is $I=1.8$ A. The current of coil is $I / 2$, because two coils are energized at the same time.

The working conditions of experimental data in Table 3: The electromagnet is Z10-T25. The parameter $a$ is $36 \mathrm{~mm}$. The other parameters of Z10-T25 are the same as the parameters of the above-mentioned simulation modeling in this paper. The coupling distances $h$ of the electromagnetic poles with the $\mathrm{p}-\mathrm{m}$ rotor are $20 \mathrm{~mm}$. The output current of the DC power source is adjusted from $I=0.55 \mathrm{~A}$ to $I=2.2 \mathrm{~A}$.

3.3.3. Comparative Analysis of the Experimental Results. The values of the load torque and driving torque of the blood pump can be obtained indirectly through the measured maximum rotating speed and the corresponding flow rate and pressure of the blood pump driven by different electromagnets. The blood pump rotating speeds under different coupling distances are given in Table 2. Hence, the law of effect of different iron core materials and structural dimensions on the driving performance of electromagnets can be reflected through the limit of rotating speed of the blood pump, as indicated in Figure 12 according to the data in Table 2.

Figure 12 indicates the following:

(1) Between D35-T25 and Z10-T25 (i.e., for the same structural dimensions and electromagnetic input parameters), the driving performance of electromagnets made with oriented silicon steels sheets has been significantly upgraded. Comparison of the load torque values corresponding to rotating speed shows that the generated load torque average value has increased for approximately $27.6 \%$ relative to the driving by electromagnets made with nonoriented silicon steel sheets.

(2) Between D35-T34 and Z10-T25, the driving performance of the electromagnets made with two different iron cores is similar. The performance of Z10 is slightly 
TABLE 3: Driving performance of the system under the condition of different current.

\begin{tabular}{|c|c|c|c|c|c|c|c|c|c|c|}
\hline Number & $\begin{array}{c}n \\
(\mathrm{r} / \mathrm{min})\end{array}$ & $I(\mathrm{~A})$ & $U(\mathrm{~V})$ & $Q(\mathrm{~L} / \mathrm{min})$ & $p_{e}(\mathrm{KPa})$ & $\begin{array}{c}\text { Output } \\
\text { power } P(\mathrm{~W})\end{array}$ & $\begin{array}{c}\text { Pressure loss } \\
p_{l}(\mathrm{KPa})\end{array}$ & $\begin{array}{l}\text { Load torque } \\
T_{L}(\mathrm{~N} \mathrm{~mm})\end{array}$ & $\begin{array}{c}\text { Driving } \\
\text { torque } T_{D} \\
(\mathrm{Nmm})\end{array}$ & $\begin{array}{c}\text { Efficiency } \eta \\
(\%)\end{array}$ \\
\hline 1 & 2000 & 0.55 & 7 & 0.43 & 0.98 & 0.01 & 0.02 & 0.049 & 0.618 & 5.6 \\
\hline 2 & 2400 & 0.60 & 8 & 0.53 & 1.31 & 0.01 & 0.04 & 0.068 & 0.674 & 7.0 \\
\hline 3 & 2800 & 0.65 & 10 & 0.62 & 1.83 & 0.02 & 0.05 & 0.095 & 0.730 & 9.1 \\
\hline 4 & 3200 & 0.70 & 12 & 0.74 & 2.15 & 0.03 & 0.07 & 0.117 & 0.786 & 10.4 \\
\hline 5 & 3600 & 0.80 & 14 & 0.87 & 3.03 & 0.04 & 0.10 & 0.172 & 0.899 & 13.4 \\
\hline 6 & 4000 & 0.85 & 17 & 1.02 & 3.85 & 0.07 & 0.13 & 0.231 & 0.955 & 17.0 \\
\hline 7 & 4400 & 0.90 & 19 & 1.19 & 4.89 & 0.10 & 0.18 & 0.312 & 1.011 & 21.6 \\
\hline 8 & 4800 & 1.00 & 21 & 1.40 & 5.77 & 0.13 & 0.25 & 0.400 & 1.123 & 24.9 \\
\hline 9 & 5200 & 1.05 & 24 & 1.59 & 6.95 & 0.18 & 0.33 & 0.506 & 1.179 & 30.0 \\
\hline 10 & 5600 & 1.10 & 27 & 1.76 & 8.40 & 0.25 & 0.40 & 0.629 & 1.235 & 35.6 \\
\hline 11 & 6000 & 1.20 & 31 & 1.95 & 9.29 & 0.30 & 0.49 & 0.723 & 1.348 & 37.5 \\
\hline 12 & 6400 & 1.25 & 35 & 2.18 & 10.78 & 0.39 & 0.61 & 0.883 & 1.404 & 44.0 \\
\hline 13 & 6800 & 1.30 & 39 & 2.41 & 11.62 & 0.47 & 0.75 & 0.998 & 1.460 & 47.8 \\
\hline 14 & 7200 & 1.40 & 43 & 2.66 & 12.76 & 0.57 & 0.91 & 1.149 & 1.572 & 51.2 \\
\hline 15 & 7600 & 1.45 & 48 & 2.93 & 13.94 & 0.68 & 1.11 & 1.320 & 1.629 & 56.7 \\
\hline 16 & 7800 & 1.50 & 51 & 3.06 & 14.21 & 0.72 & 1.21 & 1.376 & 1.685 & 57.2 \\
\hline 17 & 8000 & 1.55 & 54 & 3.14 & 15.22 & 0.80 & 1.27 & 1.473 & 1.741 & 59.2 \\
\hline 18 & 8200 & 1.60 & 57 & 3.30 & 15.64 & 0.86 & 1.41 & 1.561 & 1.797 & 60.8 \\
\hline 19 & 8400 & 1.65 & 60 & 3.44 & 15.91 & 0.91 & 1.53 & 1.625 & 1.853 & 61.4 \\
\hline 20 & 8600 & 1.70 & 64 & 3.57 & 16.27 & 0.97 & 1.64 & 1.691 & 1.909 & 62.0 \\
\hline 21 & 8800 & 1.75 & 67 & 3.76 & 16.52 & 1.04 & 1.82 & 1.783 & 1.966 & 63.5 \\
\hline 22 & 9000 & 1.80 & 69 & 3.91 & 16.94 & 1.10 & 1.97 & 1.869 & 2.022 & 64.7 \\
\hline 23 & 9200 & 1.90 & 75 & 4.02 & 17.51 & 1.17 & 2.07 & 1.946 & 2.134 & 63.9 \\
\hline 24 & 9400 & 1.95 & 80 & 4.12 & 17.96 & 1.23 & 2.19 & 2.019 & 2.190 & 64.2 \\
\hline 25 & 9600 & 2.05 & 86 & 4.22 & 18.68 & 1.31 & 2.31 & 2.104 & 2.302 & 63.8 \\
\hline 26 & 9800 & 2.15 & 90 & 4.33 & 19.34 & 1.40 & 2.41 & 2.181 & 2.415 & 63.2 \\
\hline 27 & 9900 & 2.20 & 94 & 4.40 & 19.68 & 1.44 & 2.50 & 2.242 & 2.471 & 63.5 \\
\hline
\end{tabular}

higher than that of D35. Comparison of the structures and shape parameters of the two electromagnetic iron cores indicates that the volume of Z10-T25 electromagnet is $48.4 \%$ smaller than that of the D35T34 electromagnet.

When all other parameters remained unchanged, the rotating speed of blood pump is only related to its load torque, output pressure (Table 3), so effect on drive performance of current can be obtained. When the parameter $a$ decreases from $44 \mathrm{~mm}$ to $36 \mathrm{~mm}$, the other system parameters are the same, and the limit speed $n$ of blood pump is increased from $8600 \mathrm{r} / \mathrm{min}$ to $9000 \mathrm{r} / \mathrm{min}$. So we should keep two electromagnet iron cores close together as much as possible.

\section{Conclusion}

(1) This paper describes operational principle of a large gap magnetic drive system, as well as the driving and control objectives and requirement of axial-flow blood pump.
(2) The system driving performance simulation and modeling methods have been improved, and the average deviation and calculation accuracy of the results obtained from the Ansoft Maxwell 3D simulation modeling method with the theoretical calculation result have been considerably upgraded.

(3) For the same iron core structure and electromagnetic output parameters, the electromagnet made with oriented silicon steel sheets has larger torque than the electromagnets made with nonoriented silicon steel sheets. By comparison of electromagnets made with nonoriented silicon steel sheets and oriented silicon steel sheets, the two electromagnets (i.e., D35-T34 and Z10-T25) have similar driving performance. The structural dimensions of D35-T34 are larger than Z10T25, which has greatly reduced the volume of the electromagnets.

\section{Competing Interests}

The authors declare that they have no competing interests. 


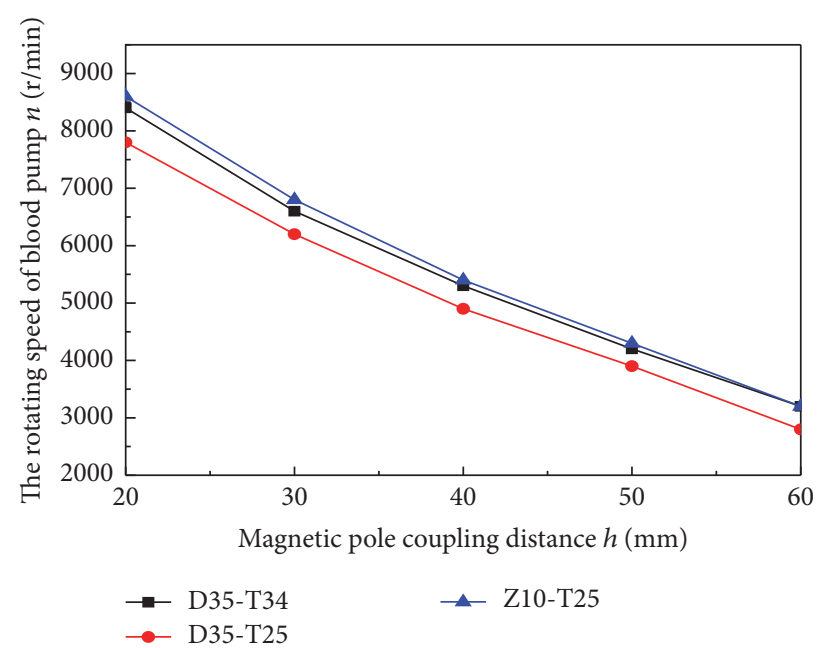

FIGURE 12: The variation law of rotational speed of blood pump with the coupling distance.

\section{Acknowledgments}

This work is supported by the National Natural Science Foundation of China (31271057), the Natural Science Foundation of Hunan Province (2016JJ6007), Hunan Provincial Department of Education Science Research Project (15A019, 16A019), and the Scientific research foundation of Changsha University.

\section{References}

[1] T. Jianping, L. Yunlong, X. Yan, L. Zhijian, Z. Zhongyan, and J. Tingting, "Dynamic characteristics of a large gap magnetic driving blood pump during start-up process," Magnetohydrodynamics, vol. 47, no. 3, pp. 283-294, 2011.

[2] H. Aminfar, M. Mohammadpourfard, and F. Ghaderi, "Twophase simulation of non-uniform magnetic field effects on biofluid (blood) with magnetic nanoparticles through a collapsible tube," Journal of Magnetism and Magnetic Materials, vol. 332, pp. 172-179, 2013.

[3] A. Aghaei, H. Khorasanizadeh, G. Sheikhzadeh, and M. Abbaszadeh, "Numerical study of magnetic field on mixed convection and entropy generation of nanofluid in a trapezoidal enclosure," Journal of Magnetism and Magnetic Materials, vol. 403, pp. 133-145, 2016.

[4] C. Yu, C. Wang, H. Deng, T. He, and B. Wei, "Magnetic field optimization and performance analysis of giant magnetostrictive actuator," Modern Manufacturing Engineering, vol. 8, pp. 136-140, 2015.

[5] A.-D. Deng, H. Zhang, and X.-J. Yang, "Parameters optimization of the strong magnetic field generation driven by electromagnetic force," Chinese Journal of High Pressure Physics, vol. 29, no. 2, pp. 123-128, 2015.

[6] J. J. Lee, C. B. Ahn, J. Choi, J. W. Park, S.-J. Song, and K. Sun, "Development of magnetic bearing system for a new thirdgeneration blood pump," Artificial Organs, vol. 35, no. 11, pp. 1082-1094, 2011.

[7] S. Jiao and D. Liu, "3D optimization design and realization of the asymmetric cusp magnetic field," Journal of Xian University of Technology, vol. 28, pp. 13-19, 2012.
[8] P. Zheng, Y. Haik, M. Kilani, and C.-J. Chen, "Force and torque characteristics for magnetically driven blood pump," Journal of Magnetism and Magnetic Materials, vol. 241, no. 2-3, pp. 292302, 2002.

[9] S. Lekhchine, T. Bahi, I. Aadlia, Z. Layate, and H. Bouzeria, "Speed control of doubly fed induction motor," Energy Procedia, vol. 74, pp. 575-586, 2015.

[10] S. Kül, O. Bilgin, and M. Mutluer, "Application of finite element method to determine the performances of the line start permanent magnet synchronous motor," Procedia-Social and Behavioral Sciences, vol. 195, pp. 2586-2591, 2015.

[11] Y. Xu, Y. Pang, and Y. Tan, "Study on the minimum energy consumption speed control method of axial flow blood pump under the condition of large gap," Magnetohydrodynamics, vol. 50, pp. 279-290, 2014.

[12] Y. Xu, J.-P. Tan, J.-F. Wang, Y.-L. Liu, and Z.-Y. Zhu, "Temperature rise of electromagnet of large-gap magnetic drive system," Journal of South China University of Technology (Natural Science Edition), vol. 41, no. 11, pp. 109-113, 2013. 


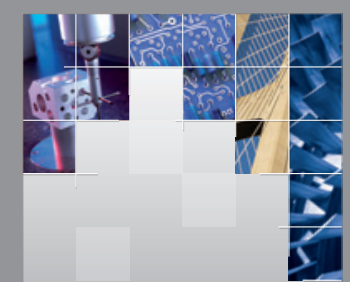

\section{Enfincering}
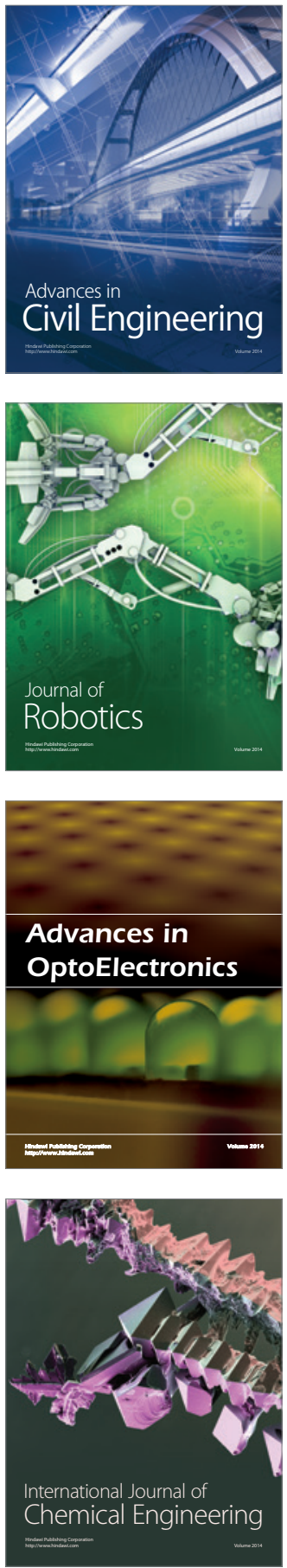

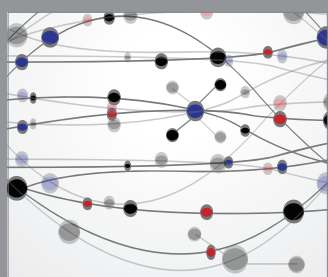

The Scientific World Journal

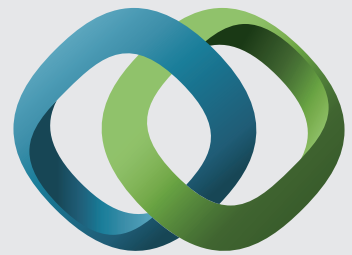

\section{Hindawi}

Submit your manuscripts at

https://www.hindawi.com
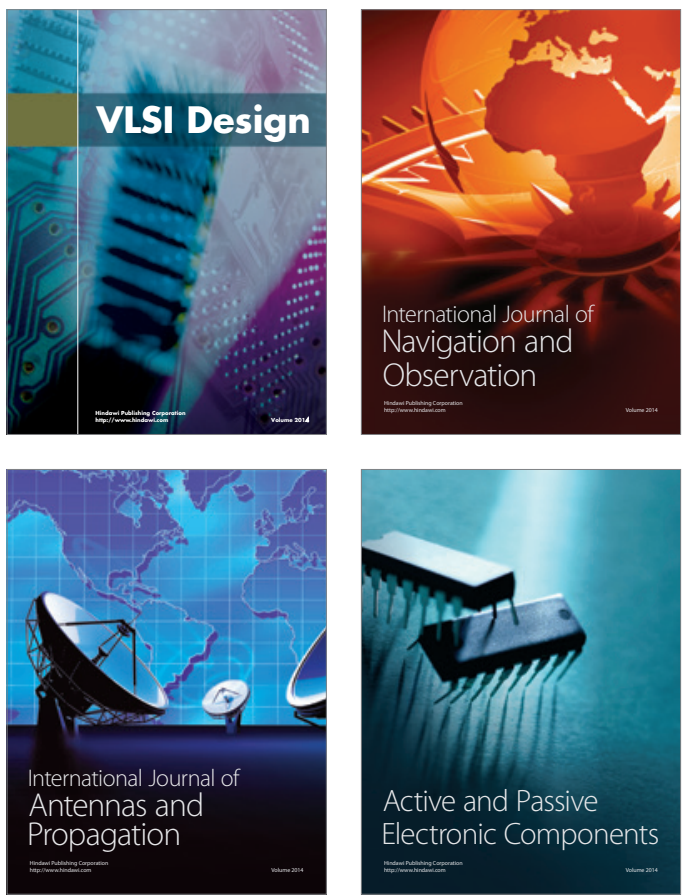
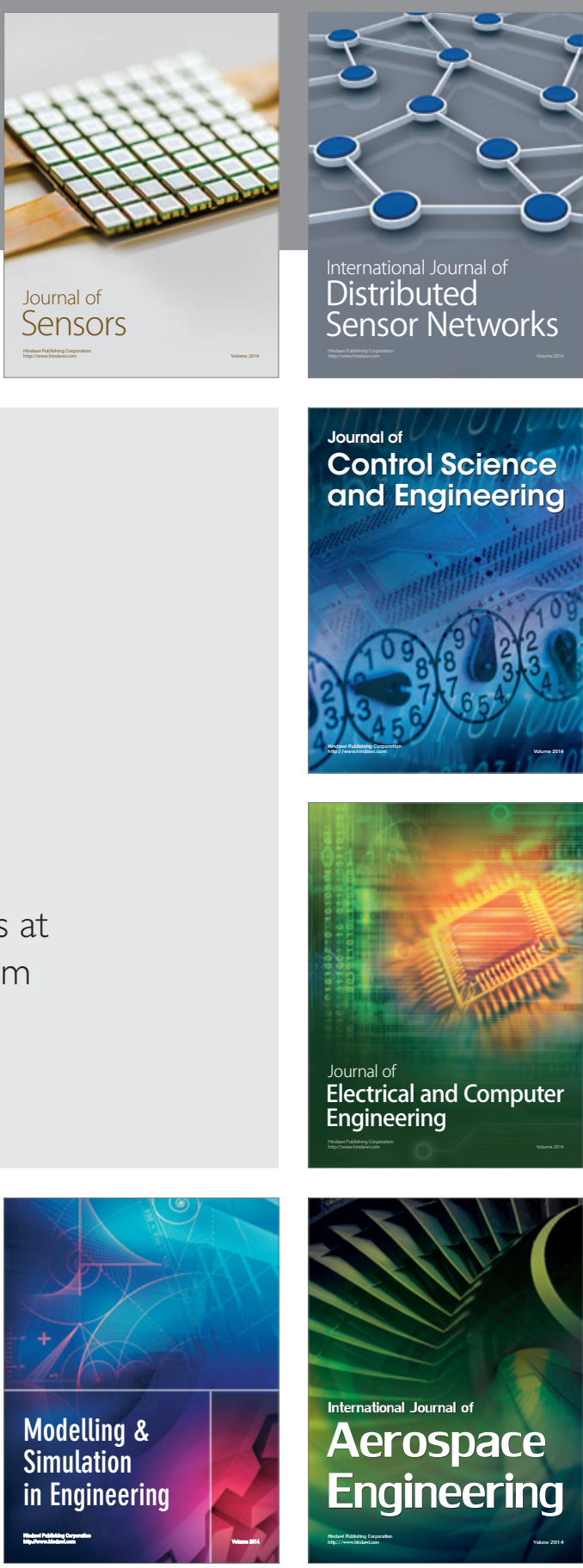

International Journal of

Distributed

Sensor Networks

$-$

Joumal of

Control Science

and Engineering
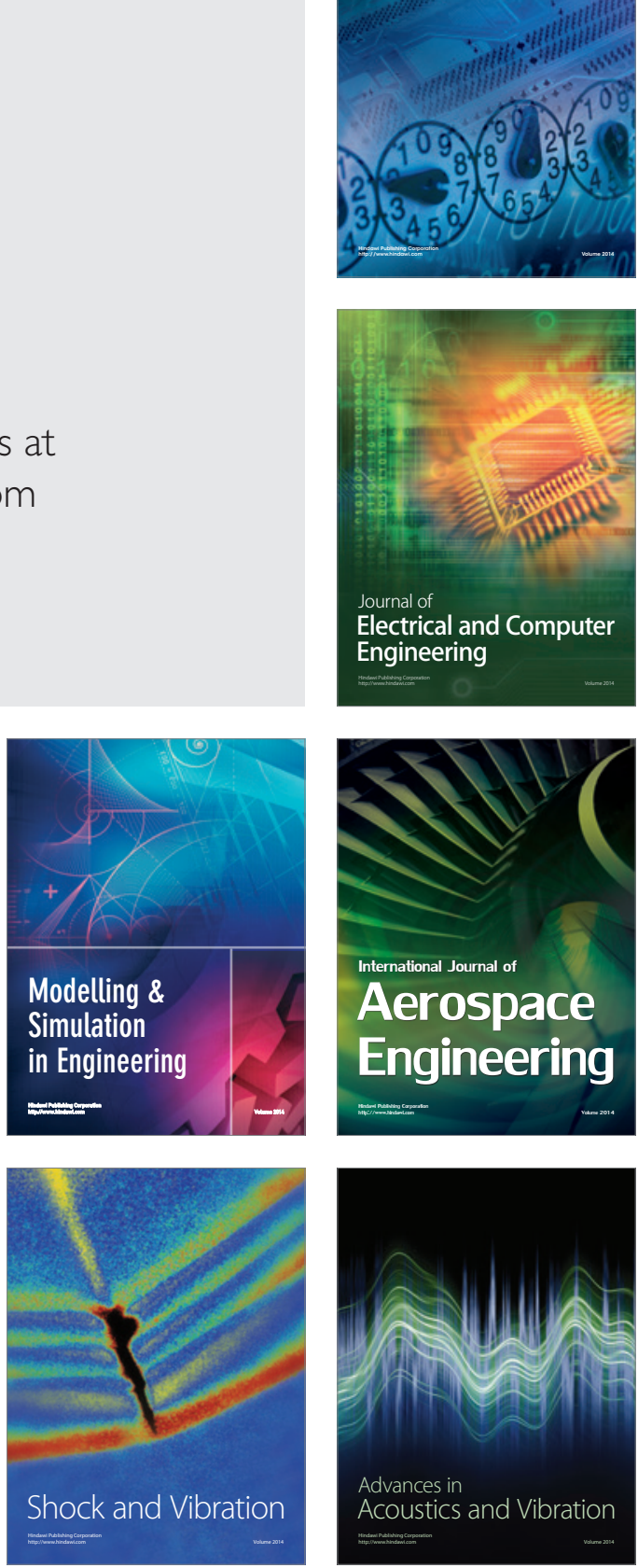